\title{
Prevalence of Activity Limitations and Association with Multimorbidity Among US Adults 50 to 64 Years Old
}

\author{
C. Barrett Bowling, MD, MSPH 1,2 , Luqin Deng, $P h D^{3}$, Swati Sakhuja, $M P H^{3}$, \\ Miriam C. Morey, $P h D^{1,2,4}$, Byron C. Jaeger, $P h D^{3}$, and Paul Muntner, $P h D^{3}$ \\ 'Durham Veterans Affairs Geriatric Research Education and Clinical Center, Durham Veterans Affairs Health Care System, Durham, NC, USA; \\ ${ }^{2}$ Divison of Geriatric Medicine, Department of Medicine, Duke University, Durham, NC, USA; ${ }^{3}$ Department of Epidemiology, University of Alabama \\ at Birmingham, Birmingham, AL, USA; ${ }^{4}$ Center for the Study of Aging/Claude D. Pepper Older Americans Independence Center (OAIC), Duke \\ University, Durham, NC, USA.
}

BACKGROUND: Functional limitations may be more common in middle-aged adults than previously recognized. However, there are few published data on the prevalence of activity limitations, and their association with multimorbidity, among adults 50 to 64 years old.

OBJECTIVE: To describe the prevalence of activity limitations and the association with multimorbidity in middleaged adults.

DESIGN: Cross-sectional analysis of US populationbased National Health and Nutrition Examination Survey (NHANES) 2011-2016.

PARTICIPANTS: The total number of communitydwelling NHANES participants aged 50-64 years old is 4217.

MAIN MEASURES: Chronic conditions included hypertension, high cholesterol, diabetes mellitus, obesity, chronic kidney disease, cancer, stroke, coronary heart disease, heart failure, chronic obstructive pulmonary disease/asthma, arthritis, and depression. Activity limitations were defined as any difficulty within each of four International Classification of Functioning (ICF) domains: functional limitations (kneeling, carrying, standing, sitting, reaching, grasping, pulling), mobility (walking 1/4 mile, climbing 10 steps), basic activities of daily living (BADLs; walking, transferring, eating, dressing), and instrumental activities of daily living (IADLs; finances, chores, cooking). We calculated prevalence ratios for activity limitations using generalized estimating equations. KEY RESULTS: The prevalence of functional limitations, mobility limitations, BADL difficulty, and IADL difficulty was $34 \%, 11 \%, 15 \%$, and $17 \%$, respectively. Seventy-two percent of participants had two or more chronic conditions; $23 \%$ had two, 18\% had three, 15\% had four, and $16 \%$ had five or more. Multivariable adjusted prevalence ratios (95\% CI) for functional limitations among those with $2,3,4$, and 5 or more chronic conditions, compared with 0-1 conditions, were 1.94 (1.43-2.63), 2.50 (1.933.23), 3.26 (2.48-4.27), and 4.54 (3.48-5.93), respectively ( $p$ trend $<0.001$ ). Larger prevalence ratios at a higher

Electronic supplementary material The online version of this article (https://doi.org/10.1007/s11606-019-05244-8) contains supplementary material, which is available to authorized users.

Received October 31, 2018

Revised May 2, 2019

Accepted July 17, 2019

Published online August 21, 2019 number of chronic conditions were present for mobility limitations, BADL difficulty, and IADL difficulty.

CONCLUSIONS: Problems with function are not limited to older adults and multimorbidity may be helpful for identifying middle-aged adults with a high prevalence of activity limitations.

J Gen Intern Med 34(11):2390-6

DOI: $10.1007 / \mathrm{s} 11606-019-05244-8$

(c) Society of General Internal Medicine (This is a U.S. government work and not under copyright protection in the U.S.; foreign copyright protection may apply) 2019

\section{INTRODUCTION}

Activity limitations, such as problems with general functional tasks, mobility limitations, and difficulty carrying out basic or instrumental activities of daily living (BADLs; IADLs), are common among adults $\geq 65$ years of age. ${ }^{1,2}$ These limitations are often considered a result, in part, of the development of chronic conditions as people age. ${ }^{3,4}$ Individual chronic conditions, such as a stroke, can cause impairments in body functions (e.g., loss of muscle function) that directly lead to activity limitations (e.g., inability to climb stairs). Multimorbidity, defined as the presence of two or more conditions that last 3 or more months, ${ }^{5,6}$ may be associated with activity limitations as impairments across multiple organ systems accumulate, reducing physiologic reserve and making one vulnerable to functional decline associated with health events.

With the increasing prevalence of obesity, diabetes, and sedentary lifestyle in early adulthood, ${ }^{7-9}$ functional problems may be more common in middle-aged adults than previously recognized. While prior studies suggest that nearly one-quarter of US adults may develop difficulty with BADLs between the ages of 50 and 64 years, ${ }^{10}$ the prevalence of activity limitations, across several domains, has not been well described in the middle-aged US population. Furthermore, while multimorbidity often arises during middle age, ${ }^{11,12}$ the association between multimorbidity and functional status has been less well studied in middle-aged adults.

The purpose of the current study was to estimate the prevalence of several domains of activity limitations described by the World Health Organization (WHO) International 
Classification of Functioning (ICF) model including general tasks (e.g., functional limitations with reaching, standing), mobility limitations (e.g., walking, climbing stairs), self-care (e.g., BADLs), and domestic life (e.g., IADLs) among US adults 50 to 64 years of age. ${ }^{1,13}$ Additionally, we aimed to evaluate the association between multimorbidity and activity limitations, by determining the prevalence of these limitations among those with a greater number of chronic conditions. To do this, we conducted a cross-sectional analysis using data from recent cycles of the National Health and Nutrition Examination Survey (NHANES), a US population-based study which enrolled middle-aged adults, and collected data on both the presence of chronic conditions and activity limitations.

\section{METHODS}

\section{Study Population}

Descriptions of the design and data collection procedures for NHANES are available online. ${ }^{14}$ NHANES is conducted in 2year cycles and uses a multistage stratified probability sampling approach to identify potential participants. Data from NHANES can be weighted to generate nationally representative prevalence estimates. The current analysis used data from NHANES 2011-2012, 2013-2014, and 2015-2016. We followed recommended procedures for pooling cycles in order to provide stable prevalence estimates. Sample weights were calculated with the sum of the weights matching the survey population at the midpoint of the 6 years used here with formulae provided by the National Center for Health Statistics. We restricted the study population to participants who were 50 to 64 years of age and completed a medical evaluation at the NHANES mobile examination center $(n=4231)$. We excluded participants who did not have information on activity limitations, including functional limitations, mobility limitations, BADLs, or IADLs $(n=14)$ resulting in a final sample of 4217 participants. The protocol for NHANES was approved by the National Center for Health Statistics (NCHS) institutional review board and all participants provided written informed consent.

\section{Data Collection}

Data for each NHANES cycle were collected through inperson participant interviews and a medical evaluation. Variables of interest in the current analysis obtained during the interview included age, gender, race/ethnicity, education, smoking status, history of chronic medical conditions, and use of medications. Height and weight were measured during the medical evaluation and were used to calculate body mass index (BMI). Three blood pressure (BP) measurements were obtained after $5 \mathrm{~min}$ of seated rest and we used the mean of these measurements to define systolic BP (SBP) and diastolic BP (DBP). Serum glucose, creatinine, and total cholesterol were measured from the blood sample collected during the study visit. Estimated glomerular filtration rate (eGFR) was calculated using the Chronic Kidney Disease Epidemiology Collaboration (CKD-EPI) equation. ${ }^{15}$ Spot urine albumin and creatinine were measured and used to calculate urinary albumin-to-creatinine ratio (ACR).

\section{Chronic Conditions and Multimorbidity}

We examined the presence of 12 common chronic conditions which included hypertension, high cholesterol, diabetes mellitus, obesity, CKD, cancer, stroke, coronary heart disease (CHD), heart failure (HF), chronic obstructive pulmonary disease (COPD)/asthma, arthritis, and depression. The selection of conditions was informed by the Centers for Disease Control and Prevention (CDC) recommendations for defining and measuring chronic conditions with modifications made based on the data available in NHANES. ${ }^{16}$ Specifically, dementia, atrial fibrillation, hepatitis, HIV, schizophrenia, autism spectrum disorders, and osteoporosis were not assessed during all NHANES cycles used in the current analysis. We chose to include obesity as a chronic condition because it is known to develop over time, be associated with poor health outcomes and functional limitations, and there are existing strategies for its prevention and treatment. ${ }^{17-20}$ We chose the CDC recommendations because we were interested in the prevalence of activity limitations among those with individual chronic conditions and by number of chronic conditions, without specifying one condition as the index condition (i.e., clinically dominant or most important) and without weighting conditions based on risk prediction models.

History of cancer, stroke, CHD, HF, COPD/asthma, and arthritis was defined based on self-report. Hypertension was defined as $\mathrm{SBP} \geq 130 \mathrm{mmHg}$ and/or DBP $\geq 80 \mathrm{mmHg}$, and/or self-reported use of antihypertensive medication. High cholesterol was defined as total cholesterol $\geq 240 \mathrm{mg} / \mathrm{dL}$ or selfreported use of a lipid-lowering medication. Diabetes mellitus was defined by a prior diagnosis, excluding during pregnancy, with concurrent use of insulin or oral glucose-lowering medication, or hemoglobin A1c $\geq 6.5 \%$. Obesity was defined as a $\mathrm{BMI} \geq 30 \mathrm{~kg} / \mathrm{m}^{2}$. Depression was defined as self-reported use of an antidepressant medication. CKD was defined as eGFR $<$ $60 \mathrm{~mL} / \mathrm{min} / 1.73 \mathrm{~m}^{2}$ or ACR $>30 \mathrm{mg} / \mathrm{g}$.

\section{Activity Limitations}

All activity limitations were assessed by self-report. In NHANES, all participants $\geq 60$ years old were asked about limitations with specific functional tasks. Activity limitations were defined as a response of "some difficulty," "much difficulty," or "unable to do," for one or more tasks. Based on the ICF model, we examined four domains of activity limitations. General functional tasks, frequently called functional limitations, included "stooping, crouching, or kneeling," "lifting or carrying something as heavy as 10 pounds," "standing up from an armless straight chair," "standing or being on your feet for about 2 hours," "sitting for about 2 hours," "reaching up over 
your head," "using your fingers to grasp or handle small objects," and "pushing or pulling large objects like a living room chair." Mobility tasks included "walking for a quarter of a mile" and "walking up 10 steps without resting." Self-care tasks were assessed with BADLs and included self-reported difficulty "walking from one room to another on the same level," "getting in or out of bed," "eating, like holding a fork, cutting food or drinking from a glass," and "dressing yourself." Domestic life activity tasks were assessed with IADLs including self-reported difficulty "managing your money," "doing chores around the house," and "preparing your own meals."

Among those $<60$ years old, activity limitations were assessed in two stages. First, participants were asked "Does a physical, mental, or emotional problem now keep you from working at a job or business?", "Are you limited in any way because of difficulty remembering or because you experience periods of confusion?", and "Are you limited in any way in any activity because of a physical, mental, or emotional problem?" Those who answered no to all three questions were considered to have no activity limitations. Participants who answered yes to one or more of these questions were then asked about the same specific activity limitations as those who were $\geq 60$ years old.

\section{Statistical Analysis}

Participant characteristics were calculated overall and separately for those with and without functional limitations, mobility limitations, BADL difficulty, and IADL difficulty. The prevalence of functional limitations, mobility limitations, BADL difficulty, and IADL difficulty was calculated for those with and without each individual chronic conditions, separately, including hypertension, high cholesterol, diabetes mellitus, obesity, CKD, cancer, stroke, CHD, HF, COPD/asthma, arthritis, and depression. Next, we calculated prevalence ratios and 95\% confidence intervals (CI) for functional limitations, mobility limitations, BADL difficulty, and IADL difficulty for those with versus without each of the individual chronic conditions. In the first model, we adjusted for age, sex, and race/ethnicity. In a second model, we further adjusted for education and cigarette smoking. In a third model, we further adjusted for the other chronic conditions (e.g., hypertension adjusted for the 11 other chronic conditions). To determine the prevalence of functional impairment among those with multimorbidity, we first calculated the percentage of participants with 0 $1,2,3,4$, and 5 or more of the 12 chronic conditions among those with versus without functional limitations. We repeated this for mobility limitations, BADL difficulty, and IADL difficulty. Finally, we calculated prevalence ratios for functional limitations, mobility limitations, BADL difficulty, and IADL difficulty for participants with $2,3,4$, and 5 or more compared with $0-1$ conditions (i.e., referent category) adjusting for age, sex, race/ethnicity, education, and cigarette smoking. We pooled those with 0 or 1 condition because of the low prevalence of 0 conditions and to provide more stable estimates. Prevalence ratios were calculated using loglinear generalized estimating equations. Linear trends were tested modeling number of conditions as a continuous variable. As prior studies of multimorbidity have not consistently included obesity as a chronic condition, we conducted a supplemental analysis removing obesity as a chronic condition of interest and calculating prevalence ratios for each of the four domains of activity limitations associated with individual chronic conditions and number of chronic conditions with adjustment for BMI in addition to the variables in models 2 and 3

Table 1 Characteristics of US Adults 50 to 64 years of age from NHANES 2011-2016

\begin{tabular}{|c|c|}
\hline Characteristic & $\begin{array}{l}\%(95 \% \text { CI }) \text { or mean } \\
(95 \% \text { CI })\end{array}$ \\
\hline Age, years & $56.7(56.5-56.9)$ \\
\hline Men, \% & $48.7(46.9-50.4)$ \\
\hline \multicolumn{2}{|l|}{ Race/ethnicity, \% } \\
\hline Non-Hispanic White & $71.1(66.7-75.5)$ \\
\hline Non-Hispanic Black & $11.3(8.8-13.9)$ \\
\hline Hispanic & $10.8(8.4-13.2)$ \\
\hline Asian & $6.8(5.3-8.3)$ \\
\hline Less than high school education, $\%$ & $14.3(12.0-16.5)$ \\
\hline Current smoking, $\%$ & $50.2(47.7-52.7)$ \\
\hline Body mass index, $\mathrm{kg} / \mathrm{m}^{2}$ & $29.9(29.5-30.3)$ \\
\hline Systolic blood pressure, $\mathrm{mmHg}$ & $126.1(125.1-127.0)$ \\
\hline Diastolic blood pressure, $\mathrm{mmHg}$ & $73.2(72.7-73.8)$ \\
\hline Antihypertensive medication use, $\%$ & $34.8(32.3-37.2)$ \\
\hline Hemoglobin A1c & $5.9(5.8-5.9)$ \\
\hline Diabetes medication use, $\%$ & $13.4(12.1-14.8)$ \\
\hline Total cholesterol, mg/dL & $202.7(200.6-204.8)$ \\
\hline Lipid-lowering therapy use, $\%$ & $27.7(25.5-29.9)$ \\
\hline $\mathrm{eGFR}, \mathrm{mL} / \mathrm{min} / 1.73 \mathrm{~m}^{2}$ & $83.7(82.4-85.0)$ \\
\hline $\mathrm{ACR}, * \mathrm{mg} / \mathrm{g}$ & $35.4(27.7-43.1)$ \\
\hline Functional limitations, $\%$ & $34.0(31.7-36.3)$ \\
\hline Mobility limitations, \% & $11.2(9.6-12.8)$ \\
\hline $\begin{array}{l}\text { Basic activities of daily living (BADLs) } \\
\text { difficulty, \% }\end{array}$ & $14.6(12.8-16.5)$ \\
\hline $\begin{array}{l}\text { Instrumental activities of daily living } \\
\text { (IADLs) difficulty, \% }\end{array}$ & $16.8(14.7-19.0)$ \\
\hline Hypertension, \% & $61.2(58.9-63.5)$ \\
\hline High cholesterol, \% & $43.8(41.5-46.1)$ \\
\hline Diabetes mellitus, \% & $16.4(14.8-17.9)$ \\
\hline Obesity, \% & $42.4(39.5-45.3)$ \\
\hline CKD, \% & $17.2(15.3-19.1)$ \\
\hline Cancer, $\%$ & $12.5(10.9-14.0)$ \\
\hline Stroke, \% & $3.1(2.4-3.7)$ \\
\hline Coronary heart disease, $\%$ & $5.3(4.4-6.2)$ \\
\hline Heart failure, $\%$ & $2.1(1.6-2.6)$ \\
\hline COPD/asthma, \% & $17.4(15.2-19.6)$ \\
\hline Arthritis, \% & $37.1(34.8-39.4)$ \\
\hline Depression, $\%$ & $17.4(15.7-19.1)$ \\
\hline \multicolumn{2}{|l|}{ Number of conditions, $\%$} \\
\hline 0 & $8.8(7.4-10.2)$ \\
\hline 1 & $18.8(17.0-20.5)$ \\
\hline 2 & $23.3(21.5-25.1)$ \\
\hline 3 & $18.2(16.7-19.8)$ \\
\hline 4 & $14.5(12.7-16.3)$ \\
\hline$\geq 5$ & $16.4(15.2-17.7)$ \\
\hline
\end{tabular}

NHANES, National Health and Nutrition Examination Survey; CI, confidence interval; eGFR, estimated glomerular filtration rate; $A C R$, albumin-to-creatinine ratio

*Geometric mean $(95 \%$ CI), depression defined as use of antidepressant 
described above. This was done both for analyses that included individual chronic conditions and for multimorbidity described above. All analyses were performed using SAS 9.4 (SAS Institute, Cary, NC) and Stata v 15 (College Station, TX).

\section{RESULTS}

\section{Participant Characteristics}

The analytic sample for these analyses included 4217 participants. Overall, participants had a mean age of 56.7 years; $48.7 \%$ were men and $71.1 \%$ were nonHispanic white (Table 1). The prevalence of functional limitations, mobility limitations, BADL difficulty, and IADL difficulty was $34.0 \%, 11.2 \%, 14.6 \%$, and $16.8 \%$, respectively. Compared with participants without activity limitations, those with limitations were older, more likely to have less than a high school education, and more likely to be taking antihypertensive and lipid-lowering medications, and had a higher mean ACR (Supplemental Table 1).

\section{Individual Chronic Conditions and Activity Limitations}

The prevalence of functional limitations, mobility limitations, BADL difficulty, and IADL difficulty was higher among those with versus without each chronic condition (Fig. 1). After multivariable adjustment, diabetes mellitus, obesity, CKD, stroke, HF, COPD/asthma, arthritis, and depression were each associated with limitations in one or more of the four domains of activity limitations (Table 2). These associations remained present when obesity was removed and multivariable adjustment included BMI (Supplemental Table 2).

\section{Multimorbidity and Activity Limitations}

Participants with more chronic conditions were progressively more likely to have activity limitations (Fig. 2). The percentage of participants with $\geq 2$ more conditions was $88 \%$ and $62 \%$ among those with versus without functional limitations, $91 \%$ and $69 \%$ among those with versus without mobility limitations, $91 \%$ and $67 \%$ among those with versus without BADL difficulty, and $90 \%$ and $67 \%$ among those with versus without IADL difficulty. After adjustment for age, sex,

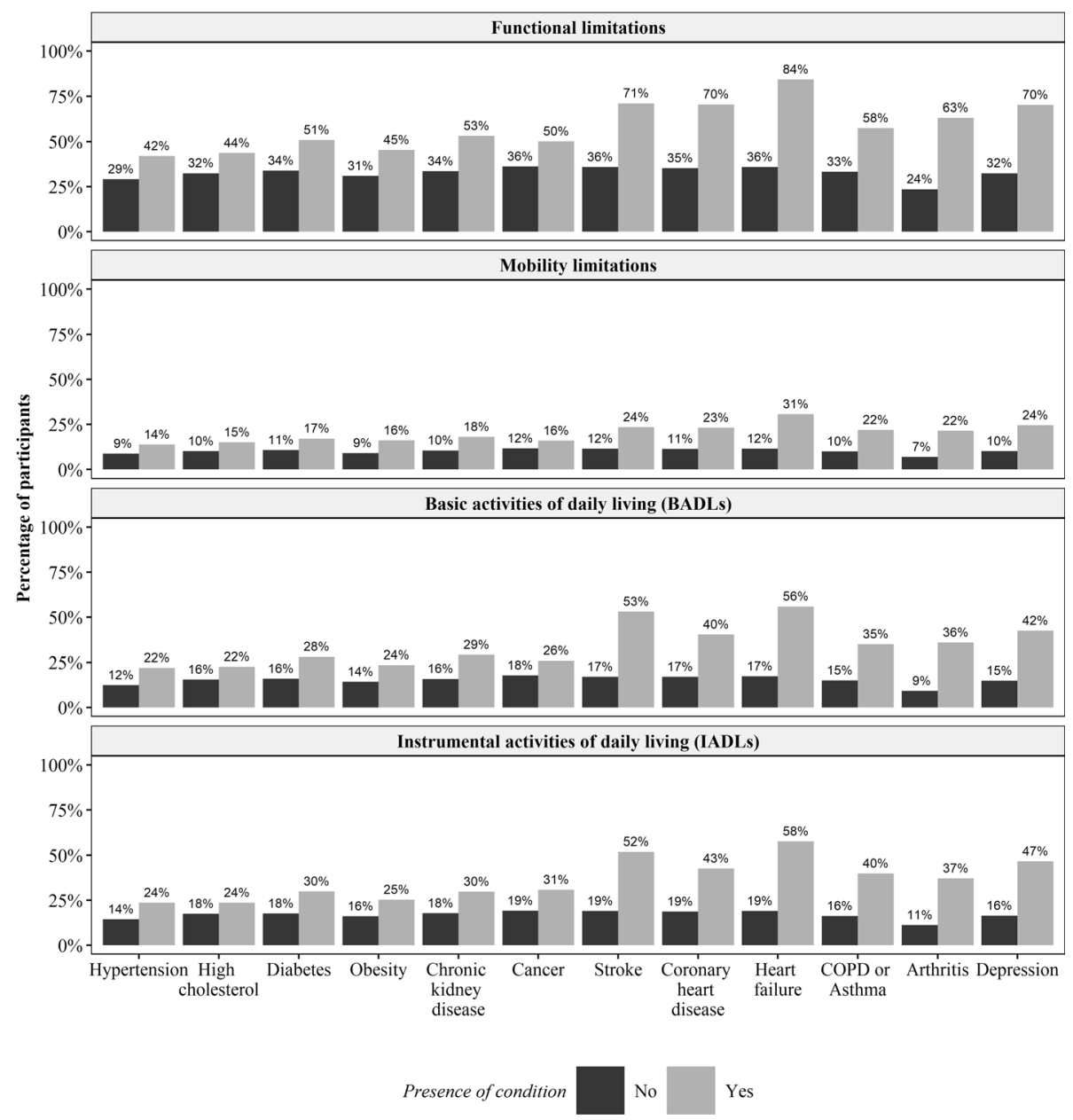

Figure 1 Prevalence of functional limitations, mobility limitations, and BADL and IADL difficulties among US adults 50 to 64 years with and without chronic conditions. 
Table 2 Prevalence Ratios (95\% CI) for Activity Limitations Among US Adults 50 to 64 Years Old with Versus Without Individual Chronic Conditions

\begin{tabular}{|c|c|c|c|c|}
\hline Chronic condition & Functional limitations & Mobility limitations & BADL difficulty & IADL difficulty \\
\hline \multicolumn{5}{|c|}{ Prevalence ratio $(95 \% \mathrm{CI})$ - model 1} \\
\hline Hypertension & $1.34(1.17-1.53)$ & $1.48(1.20-1.81)$ & $1.65(1.30-2.09)$ & $1.51(1.21-1.87)$ \\
\hline High cholesterol & $1.25(1.10-1.42)$ & $1.39(1.04-1.85)$ & $1.55(1.30-1.85)$ & $1.35(1.13-1.60)$ \\
\hline Diabetes mellitus & $1.48(1.33-1.66)$ & $1.72(1.35-2.20)$ & $1.82(1.50-2.22)$ & $1.91(1.55-2.35)$ \\
\hline Obesity & $1.67(1.43-1.94)$ & $2.00(1.56-2.57)$ & $1.83(1.43-2.35)$ & $1.75(1.36-2.26)$ \\
\hline CKD & $1.47(1.28-1.67)$ & $1.72(1.25-2.36)$ & $1.76(1.49-2.08)$ & $1.63(1.32-2.02)$ \\
\hline Cancer & $1.11(0.96-1.29)$ & $1.35(1.05-1.75)$ & $1.31(0.98-1.76)$ & $1.40(1.10-1.78)$ \\
\hline Stroke & $1.98(1.69-2.33)$ & $2.03(1.27-3.26)$ & $3.07(2.48-3.81)$ & $2.69(2.04-3.55)$ \\
\hline Coronary heart disease & $1.95(1.70-2.25)$ & $2.01(1.38-2.94)$ & $2.36(1.78-3.12)$ & $2.33(1.77-3.07)$ \\
\hline Heart failure & $2.22(1.97-2.50)$ & $2.92(1.92-4.42)$ & $3.31(2.40-4.58)$ & $3.07(2.35-4.00)$ \\
\hline COPD/asthma & $1.63(1.45-1.83)$ & $2.27(1.84-2.80)$ & $2.40(2.00-2.87)$ & $2.46(2.01-3.02)$ \\
\hline Arthritis & $2.72(2.33-3.18)$ & $3.19(2.32-4.39)$ & $4.46(3.36-5.92)$ & $3.52(2.69-4.59)$ \\
\hline Depression & $2.08(1.81-2.39)$ & $2.05(1.36-3.10)$ & $3.02(2.60-3.50)$ & $2.86(2.42-3.39)$ \\
\hline \multicolumn{5}{|c|}{ Prevalence ratio $(95 \% \mathrm{CI})$ - model 2} \\
\hline Hypertension & $1.31(1.15-1.49)$ & $1.42(1.16-1.73)$ & $1.58(1.25-2.01)$ & $1.45(1.17-1.81)$ \\
\hline High cholesterol & $1.26(1.11-1.42)$ & $1.39(1.05-1.85)$ & $1.56(1.32-1.85)$ & $1.36(1.15-1.60)$ \\
\hline Diabetes mellitus & $1.47(1.33-1.64)$ & $1.71(1.35-2.16)$ & $1.81(1.50-2.19)$ & $1.90(1.55-2.33)$ \\
\hline Obesity & $1.76(1.51-2.04)$ & $2.15(1.72-2.68)$ & $1.99(1.56-2.53)$ & $1.89(1.48-2.41)$ \\
\hline CKD & $1.40(1.24-1.59)$ & $1.61(1.16-2.24)$ & $1.64(1.40-1.91)$ & $1.53(1.24-1.87)$ \\
\hline Cancer & $1.12(0.96-1.30)$ & $1.37(1.05-1.78)$ & $1.32(0.99-1.76)$ & $1.40(1.11-1.76)$ \\
\hline Stroke & $1.73(1.49-2.01)$ & $1.63(1.01-2.62)$ & $2.51(1.95-3.23)$ & $2.21(1.66-2.95)$ \\
\hline Coronary heart disease & $1.71(1.49-1.97)$ & $1.64(1.06-2.54)$ & $1.95(1.45-2.60)$ & $1.93(1.46-2.53)$ \\
\hline Heart failure & $2.04(1.78-2.33)$ & $2.52(1.54-4.14)$ & $2.94(1.97-4.40)$ & $2.74(1.97-3.81)$ \\
\hline COPD/asthma & $1.52(1.34-1.71)$ & $2.06(1.67-2.53)$ & $2.17(1.83-2.58)$ & $2.24(1.82-2.75)$ \\
\hline Arthritis & $2.62(2.24-3.07)$ & $2.99(2.17-4.12)$ & $4.21(3.17-5.59)$ & $3.31(2.53-4.34)$ \\
\hline Depression & $1.99(1.76-2.25)$ & $1.93(1.29-2.88)$ & $2.81(2.42-3.25)$ & $2.68(2.25-3.18)$ \\
\hline \multicolumn{5}{|c|}{ Prevalence ratio $(95 \% \mathrm{CI})$ - model 3} \\
\hline Hypertension & $1.02(0.90-1.15)$ & $1.04(0.85-1.26)$ & $1.12(0.90-1.38)$ & $1.07(0.85-1.34)$ \\
\hline High cholesterol & $1.00(0.87-1.14)$ & $1.07(0.82-1.41)$ & $1.10(0.90-1.36)$ & $0.97(0.81-1.16)$ \\
\hline Diabetes mellitus & $1.10(0.98-1.23)$ & $1.18(0.90-1.55)$ & $1.24(1.02-1.50)$ & $1.39(1.16-1.68)$ \\
\hline Obesity & $1.40(1.19-1.65)$ & $1.68(1.35-2.09)$ & $1.37(1.05-1.81)$ & $1.30(1.00-1.70)$ \\
\hline CKD & $1.20(1.05-1.38)$ & $1.27(0.89-1.80)$ & $1.27(1.05-1.54)$ & $1.21(0.96-1.52)$ \\
\hline Cancer & $0.96(0.82-1.13)$ & $1.16(0.86-1.56)$ & $1.11(0.87-1.43)$ & $1.19(0.94-1.49)$ \\
\hline Stroke & $1.30(1.10-1.54)$ & $1.20(0.76-1.87)$ & $1.62(1.19-2.20)$ & $1.38(1.04-1.84)$ \\
\hline Coronary heart disease & $1.15(0.94-1.40)$ & $0.98(0.64-1.51)$ & $0.94(0.60-1.47)$ & $1.07(0.74-1.55)$ \\
\hline Heart failure & $1.21(1.01-1.44)$ & $1.37(0.79-2.39)$ & $1.58(0.94-2.65)$ & $1.41(0.90-2.23)$ \\
\hline COPD/Asthma & $1.16(1.02-1.31)$ & $1.57(1.20-2.07)$ & $1.48(1.19-1.85)$ & $1.58(1.27-1.97)$ \\
\hline Arthritis & $2.21(1.86-2.63)$ & $2.32(1.58-3.38)$ & $3.13(2.28-4.28)$ & $2.56(1.89-3.48)$ \\
\hline Depression & $1.51(1.37-1.67)$ & $1.37(0.97-1.93)$ & $1.91(1.58-2.32)$ & $1.87(1.50-2.32)$ \\
\hline
\end{tabular}

BADLs, basic activities of daily living; IADLs, instrumental activities of daily living; CI, confidence interval; CKD, chronic kidney disease; COPD, chronic obstructive pulmonary disease

Model 1: adjusted for age, sex, and race/ethnicity

Model 2: adjusted for age, sex, racelethnicity, education, and cigarette smoking

Model 3: adjusted for age, sex, racelethnicity, education, and cigarette smoking and the other chronic conditions listed in the table Italics indicates statistical significance $(P<0.05)$

race/ethnicity, education, and cigarette smoking, prevalence ratios $(95 \% \mathrm{CI})$ for functional limitations were 1.94 (1.432.63), 2.50 (1.93-3.23), 3.26 (2.48-4.27), and 4.54 (3.485.93) among those with $2,3,4$, and 5 or more versus $0-1$ chronic conditions, respectively ( $p$ trend $<0.001$; Fig. 3). Having more chronic conditions was associated with progressively higher prevalence ratios for mobility limitations, BADL difficulty, and IADL difficulty (Fig. 3). This association was also present when obesity was not included as a chronic condition and BMI was included in the multivariable adjusted model (Supplemental Table 3).

\section{DISCUSSION}

Among US adults 50 to 64 years of age, activity limitations defined by difficulty with general functional tasks, mobility limitations, BADL difficulty, and IADL difficulty were common. While the activity limitations studied here are less common than have been reported for older adults, the prevalence ranged from more than $11 \%$ of middle-aged adults reporting problems with mobility up to $34 \%$ with functional limitations, indicating a substantial burden in this population. Additionally, several individual chronic conditions were associated with activity limitations and there was a graded association between a higher number of chronic conditions with an increased prevalence of activity limitations.

Although prevalence of activity limitations has not been extensively studied in middle age, one cross-sectional analysis of those 50 to 64 years of age in the English Longitudinal Study of Aging (ELSA) found that difficulty with BADLs and mobility occurred in $15 \%$ and $18 \%$ of participants, respective$1 y .{ }^{21}$ A recent analysis of the Health and Retirement Study (HRS) found that nearly one-quarter of participants developed difficulty with one or more BADLs during middle age. ${ }^{10}$ Data on functional limitations and IADLs used in the current study 


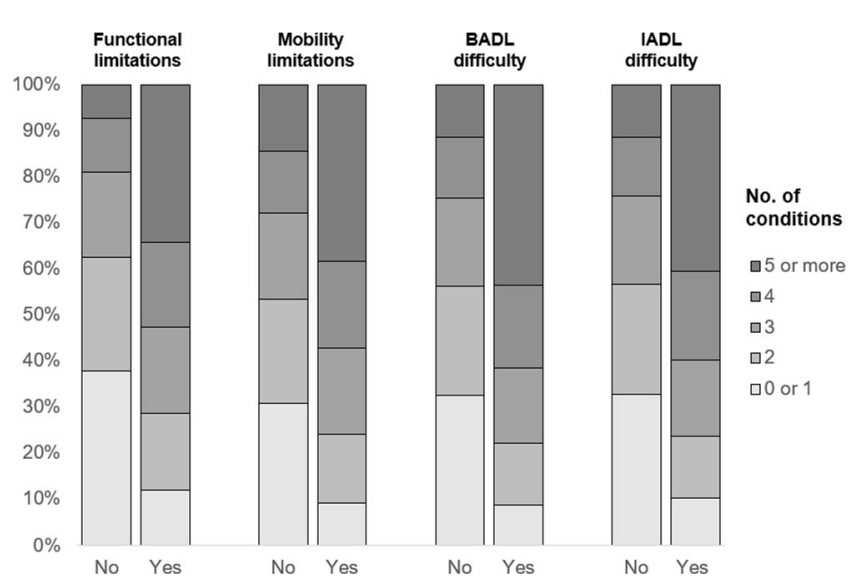

Figure 2 Percent of US adults 50 to 64 years old with multimorbidity among those with and without functional limitations, mobility limitations, basic activity of daily living (BADL) difficulty, and instrumental activity of daily living (IADL) difficulty.

provides information on domains of activity limitations (general tasks, domestic life) that have not been as well studied in this age range.

Activity limitations are often considered a part of the aging process and functional assessment is a part of geriatrics care. ${ }^{22}$ However, the limitations we studied were common among those 50 to 64 years old, and markedly so for basic functional tasks with movements that included kneeling, carrying, standing, sitting, reaching, grasping, and pulling which occurred in more than one-third of the study population. The higher prevalence of functional limitations (34\%) compared with IADL difficulty (17\%) and BADL difficulty $(15 \%)$ is consistent with the conceptualization of a hierarchical nature of functional loss. Because the functional limitations studied here represent "building blocks" of function, loss of which can lead to disability, identifying this process in middle age may be one step towards slowing disablement. ${ }^{23}$ These findings are consistent with prior work in the Physical Performance Across the Life Span (PALS) study, in which both declines in direct measures of activity limitations (i.e., gait speed, balance, chair stands) and changes in immune and metabolic biomarkers associated with poor function in older age began to occur in middle age. ${ }^{24,}{ }^{25}$ While routine assessment of function in middle age may be warranted, focusing on IADLs and BADLs, as is often done in Geriatrics practice, may miss early activity limitations in middle-aged adults that could be addressed before they impact patients' life participation.

In the current study, activity limitations co-occurred with several chronic conditions including diabetes, obesity, CKD, stroke, CHD, COPD/asthma, arthritis, and depression. Additionally, there was a graded association between a higher number of chronic conditions and an increased prevalence of activity limitations, suggesting that multimorbidity may be a useful marker of activity limitations among middle-aged adults. Information on the prevalence of chronic conditions is often available in electronic health records and could be used to identify adults 50 to 64 years old with multimorbidity for subsequent functional assessment. Future studies should determine how to practically implement functional assessment into routine care for this population given shortages of geriatricians and competing demands faced by primary care providers. Additional studies may be useful to determine how and when to intervene to prevent functional decline in middle-aged adults.

Strengths of the current analysis included the ability to generate nationally representative estimates with data available on multiple domains of activity limitation and data on the presence of multiple chronic conditions. However, data on physical performance limitations, such as slow gait speed, which have been shown to be common in middle age, ${ }^{24}$ or measures of observed function were not available. Information on some activity limitations (e.g., bathing difficulty) were not assessed. We relied on self-reports of activity limitations, without corroboration from family members, direct clinical observation, or assessment of cognitive function. Therefore, misclassification of function was possible. Data on some chronic conditions that are included in the CDC recommendations, such as dementia, atrial fibrillation, and osteoporosis, were not assessed in the NHANES cycles that we analyzed. However, because of the low prevalence of these conditions in middle age, it is unlikely that inclusion of these conditions would have changed the overall conclusions of the current study. We may have overestimated the prevalence of cancer because we were not able to identify and omit non-melanoma skin cancer. Additionally, we were not able to study incident activity limitations or temporal associations between multimorbidity and function, which may be important as limitations have been shown to be associated with development of some chronic conditions. ${ }^{26}$

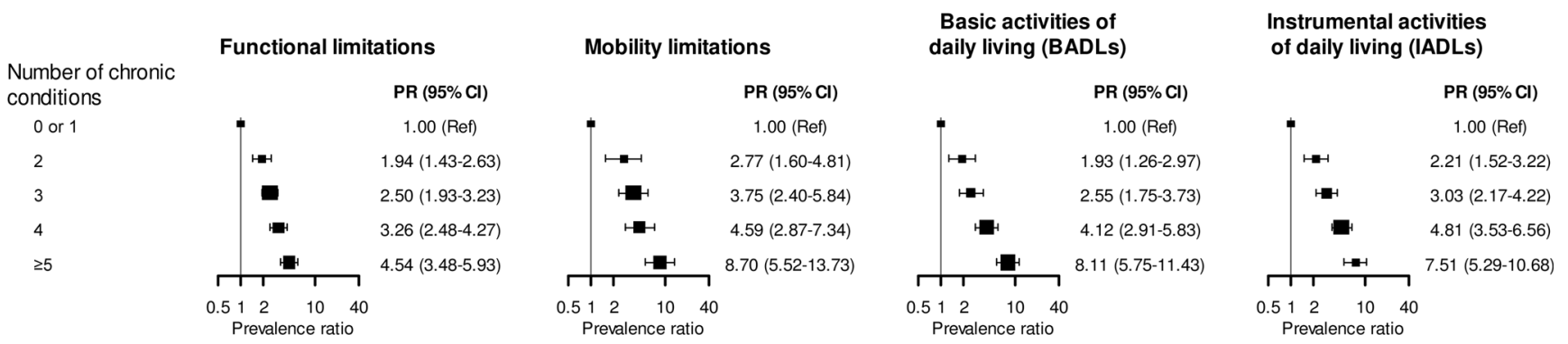

Figure 3 Prevalence ratios (95\% CI) for the association of number of chronic conditions with functional limitations, mobility limitations, BADL difficulty, and IADL difficulty among US adults 50 to 64 years old. Prevalence ratios adjusted for age, sex, race/ethnicity, and cigarette smoking. $p$ trend for all models, $<0.001$. 
Because function is dynamic with episodes of limitations and recovery, ${ }^{27}$ one-time assessment of function may overestimate the prevalence of activity limitations or miss activity limitations that were important, but resolved prior to participation in the study. We were not able to determine the impact of early adulthood income and financial resources on the association between multimorbidity and activity limitations. Although arthritis was included as a chronic condition, data on pain related to chronic arthritis were not available. Limitations of the current analysis highlight knowledge gaps in existing literature that could be addressed in future prospective studies that include identification of risk factors in early adulthood for activity limitations and assess the role of physical performance measurement for defining activity limitations in middle age.

In conclusion, activity limitations, defined as limitations in general functional tasks, mobility limitations, BADL difficulty, and IADL difficulty, are common among US adults 50 to 64 years of age. Additionally, the prevalence of activity limitations was markedly higher among individuals with progressively more chronic conditions. The findings from the current study suggest that problems with function are not limited to older adults and multimorbidity may be an important marker for identifying middle-aged adults at highest risk for prevalent activity limitations. Routine assessment for activity limitations, especially among those with multimorbidity, may identify middle-aged adults who would benefit from clinical care that addresses risk factors for functional limitations and engages family and community support services to prevent decline.

Funding Source: Support was provided through the National Heart, Lung, and Blood Institute (R01HL133618) to Dr. Bowling. Dr. Morey's contributions were further supported by the Duke Pepper Older Americans Independence Center (P3OAG028716). This work was also supported by the Durham Center of Innovation to Accelerate Discovery and Practice Transformation (ADAPT) (CIN 13-410) at the Durham VA Health Care System.

Corresponding Author: C. Barrett Bowling, MD, MSPH; Department of EpidemiologyUniversity of Alabama at Birmingham, Birmingham, AL, USA (e-mail: barrett.bowling@duke.edu).

\section{Compliance with Ethical Standards:}

Conflict of Interest: The authors declare that they have no conflict of interest.

Disclaimer/Disclosures: The views expressed here/in this manuscript are those of the authors and do not necessarily represent the views of the National Heart, Lung, and Blood Institute; the National Institutes of Health; or the Department of Health and Human Services or Department of Veterans Affairs.

\section{REFERENCES}

1. Jette AM. Toward a common language of disablement. J Gerontol A Biol Sci Med Sci. 2009;64(11):1165-1168.

2. Seeman TE, Merkin SS, Crimmins EM, Karlamangla AS. Disability trends among older Americans: National Health And Nutrition Examination Surveys, 1988-1994 and 1999-2004. Am J Public Health. 2010;100(1):100-107.
3. Fried LP, Guralnik JM. Disability in older adults: evidence regarding significance, etiology, and risk. J Am Geriatr Soc. 1997;45(1):92-100.

4. Verbrugge LM, Jette AM. The disablement process. Soc Sci Med. 1994;38(1): 1-14.

5. American Geriatrics Society Expert Panel on the Care of Older Adults with M. Patient-centered care for older adults with multiple chronic conditions: a stepwise approach from the American Geriatrics Society: American Geriatrics Society Expert Panel on the Care of Older Adults with Multimorbidity. J Am Geriatr Soc. 2012;60(10):1957-1968.

6. Diederichs C, Berger K, Bartels DB. The measurement of multiple chronic diseases-a systematic review on existing multimorbidity indices. $J$ Gerontol A Biol Sci Med Sci. 2011;66(3):301-311.

7. An R, Xiang X, Yang Y, Yan H. Mapping the prevalence of physical inactivity in U.S. States, 1984-2015. PLOS One. 2016;11(12):e0168175.

8. Flegal KM, Carroll MD, Ogden CL, Curtin LR. Prevalence and trends in obesity among US adults, 1999-2008. JAMA. 2010;303(3):235-241.

9. Menke A, Casagrande S, Geiss L, Cowie CC. Prevalence of and trends in diabetes among adults in the United States, 1988-2012. JAMA. 2015;314(10):1021-1029.

10. Brown RT, Diaz-Ramirez LG, Boscardin WJ, Lee SJ, Steinman MA. Functional impairment and decline in middle age: a cohort study. Ann Intern Med. 2017;167(11):761-768.

11. Barnett K, Mercer SW, Norbury M, Watt G, Wyke S, Guthrie B. Epidemiology of multimorbidity and implications for health care, research, and medical education: a cross-sectional study. Lancet. 2012;380(9836):37-43.

12. Bowling CB, Plantinga L, Phillips LS, et al. Association of multimorbidity with mortality and healthcare utilization in chronic kidney disease. J Am Geriatr Soc. 2017;65(4):704-711.

13. World Helath Organization. Towards a common language for functioning, disability, and health, ICF, The international classification of functioning, disability and health. In. Geneva, Switzerland: World Health Organization; 2002.

14. National Center for Health Statistics. National Health and Nutrition Examination Survey: Center for Disease Control and Prevention. 2018; http://www.cdc.gov/nchs/nhanes.htm. Accessed May 31, 2019

15. Levey AS, Stevens LA, Schmid $\mathrm{CH}$, et al. A new equation to estimate glomerular filtration rate. Ann Intern Med. 2009;150(9):604-612.

16. Goodman RA, Posner SF, Huang ES, Parekh AK, Koh HK. Defining and measuring chronic conditions: imperatives for research, policy, program, and practice. Prev Chronic Dis. 2013;10:E66.

17. Bray GA, Kim KK, Wilding JPH, World Obesity F. Obesity: a chronic relapsing progressive disease process. A position statement of the World Obesity Federation. Obes Rev. 2017;18(7):715-723.

18. Martin DW. House of Delegates. Resolution 420. Recognition of Obesity as a Disease. American Medical Assocation. 2013. Available at: https:// www.ama-assn.org/sites/ama-assn.org/files/corp/media-browser/public/hod/a13-reference-committee-reports_0.pdf Accessed May 31, 2019

19. Kilov D, Kilov G. Philosophical determinants of obesity as a disease. Obes Rev. 2018;19(1):41-48.

20. Vasquez E, Batsis JA, Germain CM, Shaw BA. Impact of obesity and physical activity on functional outcomes in the elderly: data from NHANES 2005-2010. J Aging Health. 2014;26(6):1032-1046.

21. Gardener EA, Huppert FA, Guralnik JM, Melzer D. Middle-aged and mobility-limited: prevalence of disability and symptom attributions in a national survey. J Gen Intern Med. 2006;21(10):1091-1096.

22. Gill TM. Geriatric medicine: it's more than caring for old people. Am J Med. 2002;113(1):85-90.

23. Guralnik JM, Ferrucci L. Assessing the building blocks of function: utilizing measures of functional limitation. AmJ Prev Med. 2003;25(3 Suppl 2):112-121.

24. Hall KS, Cohen HJ, Pieper CF, et al. Physical performance across the adult life span: correlates with age and physical activity. J Gerontol A Biol Sci Med Sci. 2017;72(4):572-578.

25. Parker D, Sloane R, Pieper CF, et al. Age-related adverse inflammatory and metabolic changes begin early in adulthood. J Gerontol A Biol Sci Med Sci. 2019;74(3):283-289. https://doi.org/10.1093/gerona/gly121

26. Bowling CB, Fonarow GC, Patel K, et al. Impairment of activities of daily living and incident heart failure in community-dwelling older adults. Eur $J$ Heart Fail. 2012;14(6):581-587.

Publisher's Note Springer Nature remains neutral with regard to jurisdictional claims in published maps and institutional affiliations. 